\title{
EBSD-based techniques for characterization of microstructural restoration processes during annealing of metals deformed to large plastic strains
}

\author{
Godfrey, A.; Mishin, Oleg; Yu, Tianbo
}

Published in:

Materials Science Forum

Link to article, DOI:

10.4028/www.scientific.net/MSF.715-716.203

Publication date:

2012

Document Version

Early version, also known as pre-print

Link back to DTU Orbit

Citation (APA):

Godfrey, A., Mishin, O., \& Yu, T. (2012). EBSD-based techniques for characterization of microstructural restoration processes during annealing of metals deformed to large plastic strains. Materials Science Forum, 715-716, 203-210. https://doi.org/10.4028/www.scientific.net/MSF.715-716.203

\section{General rights}

Copyright and moral rights for the publications made accessible in the public portal are retained by the authors and/or other copyright owners and it is a condition of accessing publications that users recognise and abide by the legal requirements associated with these rights.

- Users may download and print one copy of any publication from the public portal for the purpose of private study or research.

- You may not further distribute the material or use it for any profit-making activity or commercial gain

- You may freely distribute the URL identifying the publication in the public portal 


\title{
EBSD-based techniques for characterization of microstructural restoration processes during annealing of metals deformed to large plastic strains
}

\author{
A. Godfrey ${ }^{1, a}$, O.V. Mishin ${ }^{2, b}$ and T.B. $\mathrm{Yu}^{2}$ \\ ${ }^{1}$ Laboratory of Advanced Materials, Department of Materials Science and Engineering, Tsinghua \\ University, Beijing 100084, China \\ ${ }^{2}$ Danish-Chinese Center for Nanometals, Materials Research Division, Risø National Laboratory \\ for Sustainable Energy, Technical University of Denmark, DK-4000 Roskilde, Denmark \\ a awgodfrey@mail.tsinghua.edu.cn; bolmi@risoe.dtu.dk
}

Keywords: recrystallization, coarsening, EBSD, ECAE, severe plastic deformation

\begin{abstract}
Some methods for quantitative characterization of the microstructures deformed to large plastic strains both before and after annealing are discussed and illustrated using examples of samples after equal channel angular extrusion and cold-rolling. It is emphasized that the microstructures in such deformed samples exhibit a heterogeneity in the microstructural refinement by high angle boundaries. Based on this, a new parameter describing the fraction of regions containing predominantly low angle boundaries is introduced. This parameter has some advantages over the simpler high angle boundary fraction parameter, in particular with regard to data collected from electron-backscatter diffraction investigations, where boundaries with very low misorientation angles cannot be reliably detected. It is shown how this parameter can be related to the recrystallization behavior. Another parameter, based on mode of the distribution of dislocation cell sizes is outlined, and it is demonstrated how this parameter can be used to investigate the uniformity, or otherwise, of the restoration processes occurring during annealing of metals deformed to large plastic strains.
\end{abstract}

\section{Introduction}

There is a considerable interest in the deformation of bulk metallic samples to large plastic strains with the objective of developing material with a fine grain size. A large number of processes to achieve such deformation have now been developed, and the benefits and advantages of each process are under continued debate [1-3]. It is typically found that although the mechanical strength can be increased as a result of the application of such deformation process, the resulting material exhibits low work hardening capability and consequently low ductility. Annealing can be used to restore the sample ductility, but only with a simultaneous loss of mechanical strength. In general therefore a balance must be sought between these two material properties [4,5].

It has been reported that for some samples deformed to high plastic strains annealing does not result in the classical discontinuous recrystallization process, but rather results in a continuous coarsening of the deformed microstructure [6,7]. Such a process is desirable as it offers greater possibilities to obtain a balance between mechanical strength and ductility. In many publications it is suggested that such continuous recrystallization is found when the deformed microstructure contains a large fraction of high angle boundaries, and thus by analogy the coarsening can be viewed as a grain growth process driven by the curvature of the deformation-induced boundaries.

It is important to remember however two important facts concerning the microstructures of metals deformed to large plastic strains. The first is that a significant fraction of low angle boundaries remain in a material even following deformation to very high plastic strains. For example, in nickel deformed by high pressure torsion to a strain of $\varepsilon_{\mathrm{vM}}=300$ approx. $30 \%$ of the boundaries were found in a transmission electron microscope study to be of less than $15^{\circ}$ 
misorientation angle [8]. The second fact is that heterogeneity is a key characteristic of all deformation microstructures. This heterogeneity can take various forms, from a variation of deformation microstructure with crystal orientation, to the presence of localized regions of enhanced deformation (e.g. shear bands and localized glide bands). The spatial distribution of the microstructural refinement during plastic deformation should therefore also be considered.

A key related point is that investigations of both the deformed microstructure and recrystallization are most conveniently carried out using electron backscatter diffraction methods, for which there is a lower limit on the misorientation angle that can be detected (typically 1.5 to $2^{\circ}$ for metals deformed to very high plastic strains). It is important therefore to take this into account when considering parameters for the characterization of the deformed microstructure and for analyzing the nature of the processes taking place during subsequent annealing treatments.

\section{Characterization of microstructural refinement.}

Figure 1a shows an orientation map constructed from EBSD measurements on a copper sample deformed by the equal channel angular extrusion (ECAE) process via route A (die angle $=120^{\circ}$ ) to a strain of 10 [9], showing boundaries of misorientation angle greater than $2^{\circ}$ and $15^{\circ}$ as thin and thick black lines respectively. For this microstructure the fraction of high angle boundaries, defined here as:

$$
\mathrm{f}_{\mathrm{HAB}}=\mathrm{L}_{\mathrm{A}}\left(>15^{\circ}\right) / \mathrm{L}_{\mathrm{A}}\left(>2^{\circ}\right)
$$

where $L_{A}(>\theta)$ is the length per area of boundary of misorientation angle greater than $\theta$, is $61 \%$. A variation in the extent of refinement by high angle boundaries can, however, be clearly seen, with some regions present where the structure clearly contains mostly low angle boundaries.

Based on this and other observations, it has been suggested [9-12] that a useful parameter for describing the extent of microstructural refinement by high angle boundaries is the fraction of the material containing predominantly only low angle misorientations (the low misorientation region fraction, or $\left.\mathrm{f}_{\mathrm{LMR}}\right)$. The procedure for calculation of this parameter is as follows. Initially a grain reconstruction is carried out using a boundary misorientation angle definition typically in the range $5-8^{\circ}$. Next the size of each detected "grain" based on this misorientation angle definition is calculated and the grains are divided into two classes (in many cases the detected grains actually consist of clusters of two or more dislocation cells). Those grains with a size greater than some chosen value are considered to be low misorientation regions (LMRs), as such regions contain predominantly only low angle misorientations, whilst the remaining material is labeled as high misorientation regions (HMRs). The result of partitioning the microstructure in this manner is illustrated in Fig. 1b, where the LMR fraction is shaded in dark grey. All of these calculations can be carried out using routines in commercially available EBSD post-processing packages.

As a result of this partitioning, several quantitative parameters can be considered to describe the microstructure. The simplest parameter is the area fraction of the LMRs, which for the map shown in Fig. 1 is $21 \%$. An important point to note is that the parameter $f_{\mathrm{LMR}}$ is not particularly sensitive to the fact that EBSD measurements on deformed samples cannot reliably detect misorientations of below $1.5-2.0^{\circ}$. This is in contrast to the conventionally used parameter of $\mathrm{f}_{\mathrm{HAB}}$, which depends explicitly on the choice of the misorientation cut-off angle (the denominator in Eqn(1)) [13]. This lack of sensitivity can be illustrated by applying a modified-Kuwahara filter in order to improve the angular resolution of the data set. An EBSD map of the data after 1 pass of a $3 \times 3$ maximum subgrid-minimum variance filter [14] with a cut-off angle of $1.7^{\circ}$ is shown in Fig. 1c, where the LMRs are shaded in grey. In the unfiltered data set, a minimum misorientation angle of $2.0^{\circ}$ is required to suppress noise in the orientation data, whereas after filtering misorientations down to less than $1.5^{\circ}$ can be detected. It can be seen that the detected LMRs in the filtered data are almost identical to those in the unfiltered data. 
(a)

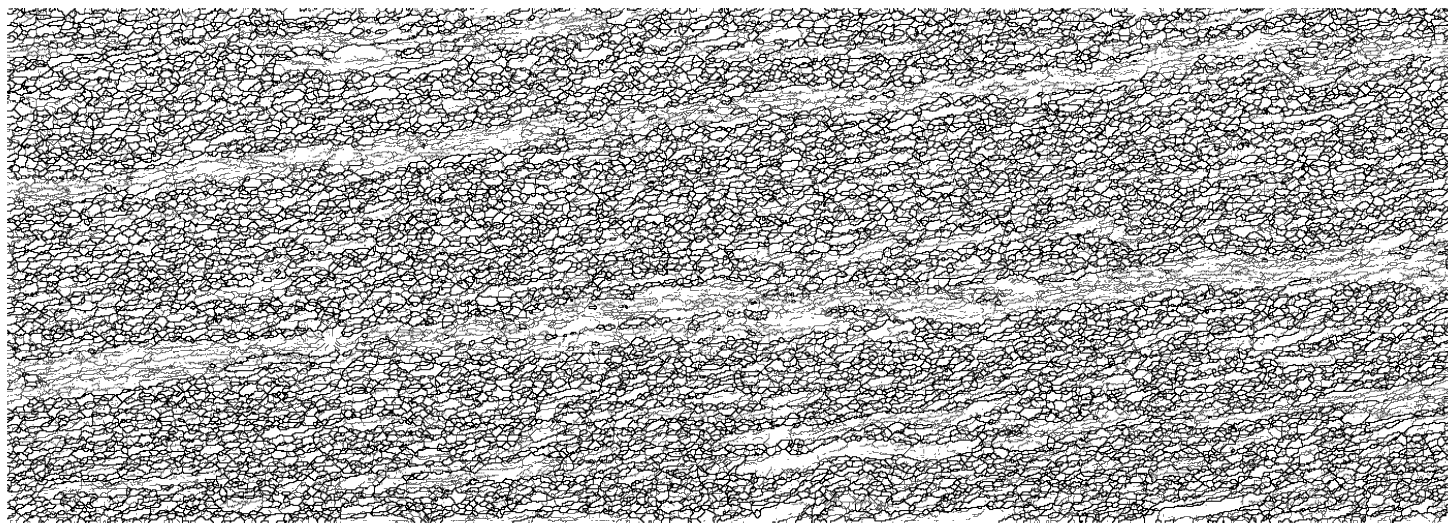

(b)
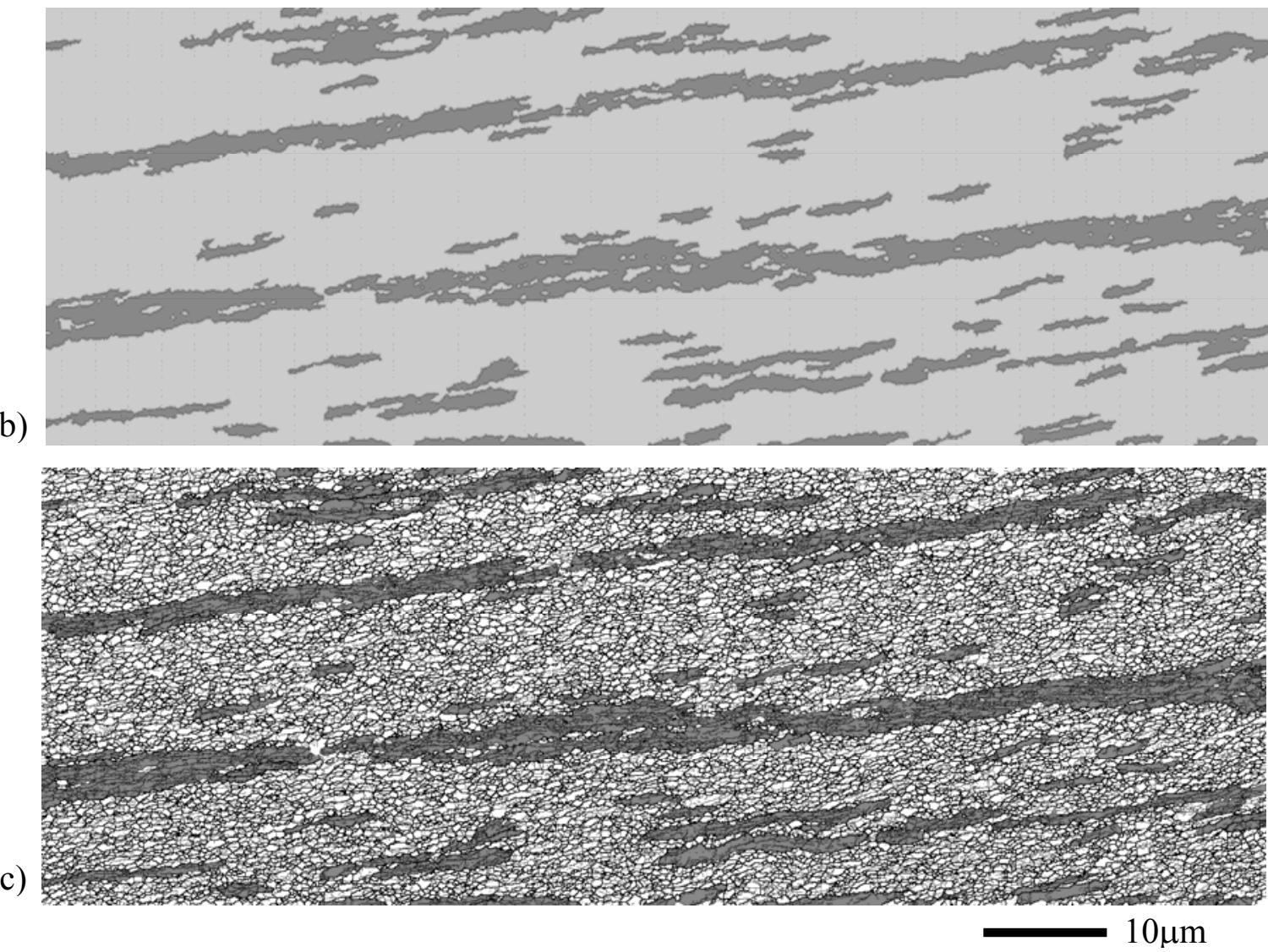

Figure 1: EBSD maps for a $\mathrm{Cu}$ sample deformed by ECAE to a strain of $\varepsilon=10$ using route $\mathrm{A}$ and a die-angle of $120^{\circ}$ : (a) boundary misorientation map; (b) partitioned into LMR (dark shading) and HMR (light shading) fractions; and (c) MSMV-filtered data set. In (a) and (c) thin black lines represent misorientations $>2^{\circ}$ and $>1.5^{\circ}$, respectively; in both maps bold black lines represent misorientations of $>15^{\circ}$.

The average misorientation and the fraction of high angle boundaries within the LMR and HMR subsets can also be calculated. The values of these parameters for the unfiltered data are given in Table 1. It is important to remember that these values are affected by the choice of the lowest reliably detectable misorientation angle as described above. In addition, the LMRs are frequently found in clusters such that the values given for the mean misorientation of the LMR subset and for $\mathrm{f}_{\mathrm{HAB}}$ of the LMR subset also include a contribution from high angle boundaries that separate adjacent LMRs.

Another advantage of the LMR/HMR partitioning method for quantification of the deformed structure is that because the method selects regions distributed throughout the examined area it is also possible to use the parameter to probe the length scale of the sample heterogeneity due to the 
variation in local refinement by high angle boundaries. The basis of the method for doing this is illustrated in Fig. 2. For the entire region investigated the value of $f_{\mathrm{LMR}}=21 \%$. If the map is divided into four quarters, then the values of $f_{L M R}$ for each quarter vary from $17 \%$ to $26 \%$. If the map is divided again into 8 regions, then a still larger variation in $f_{\mathrm{LMR}}$ is found. As smaller and smaller regions are probed, a distribution of values of $f_{L M R}$ will be obtained, so that the standard deviation of $\mathrm{f}_{\mathrm{LMR}}$ can be calculated as a function of the region size. A length scale can then be defined by taking the region size that gives a specific relative standard deviation (defined as the standard deviation divided by the mean). In practice, rather than subdividing the structure in this way, testgrids of varying size can be applied to the data set. Details of the calculation procedure are also given elsewhere [12].
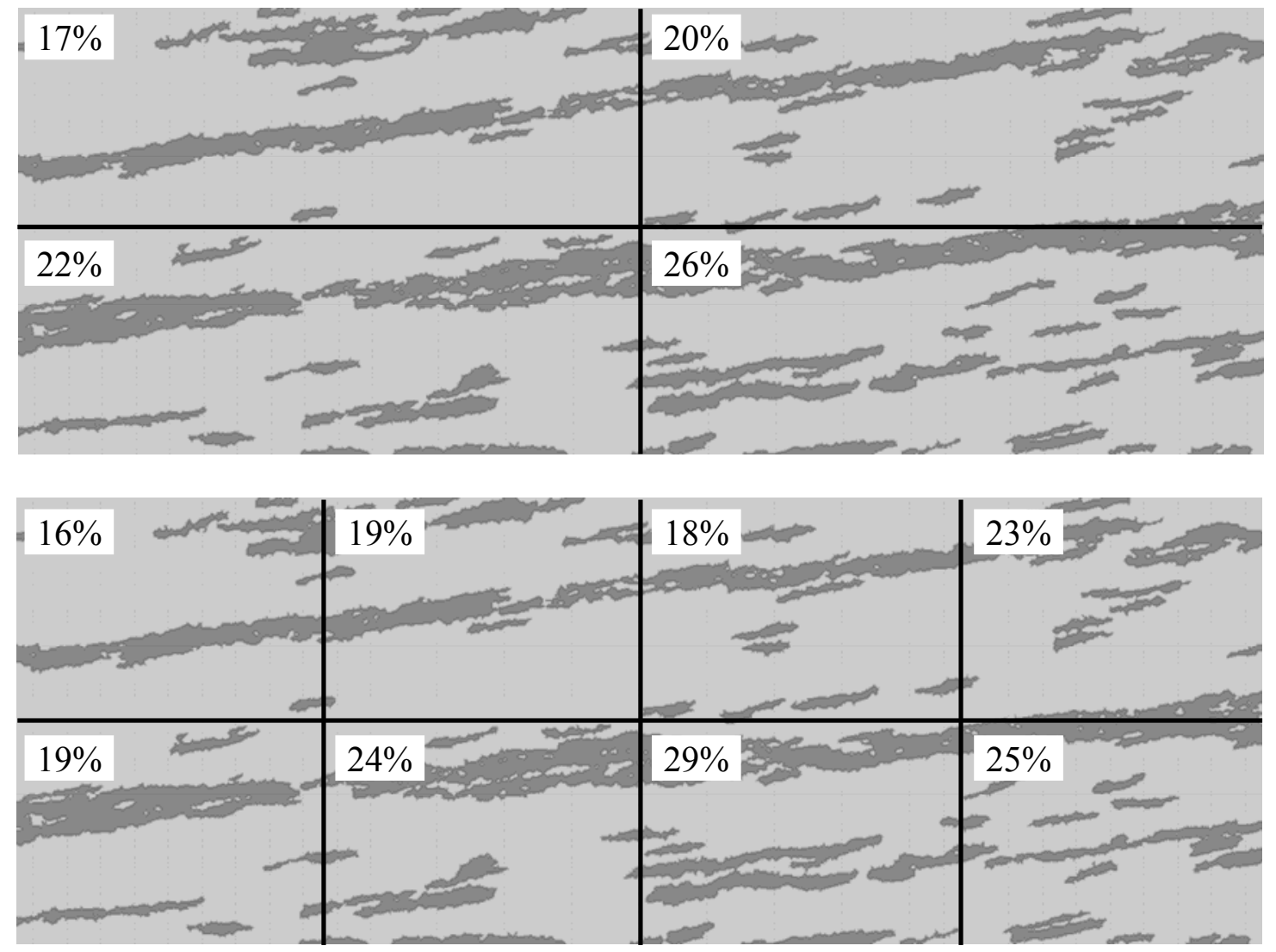

Fig. 2: Illustration of the approach taken for investigation of the length scale associated with heterogeneity in refinement by high angle boundaries. The value of $f_{\text {LMR }}$ for each region is indicated.

For a given EBSD map, both the value $\mathrm{f}_{\mathrm{LMR}}$ and the characteristic length scale of this parameter will vary with the chosen values of $\theta^{*}$ and $A^{*}$ used to partition the microstructure. Some example data are given in Fig. 3, which shows the variation in $f_{\mathrm{LMR}}$ as a function of $\mathrm{A}^{*}$ for fixed value of $\theta^{*}$ of $5^{\circ}$ and $7^{\circ}$ and as a function of $\theta^{*}$ for a fixed value of $A^{*}$ of $2.5 \mu \mathrm{m}^{2}$. As expected, $\mathrm{f}_{\mathrm{LMR}}$ increases with increasing $\theta^{*}$, and with decreasing $A^{*}$. The value of $f_{\mathrm{LMR}}$ varies almost linearly with $\theta^{*}$. A value of $5^{\circ}$ is suggested as a value above the EBSD detection limit, but still well below the value of $15^{\circ}$ conventionally used to define a high angle boundary. The value of $f_{\text {LMR }}$ varies strongly with $A^{*}$ and more care is needed to select an appropriate value for this parameter. One way to fix the value of $A^{*}$ is to take the value as a fixed multiple of $d_{i}^{2}$, where $d_{i}$ is the linear intercept spacing obtained using the lowest possible misorientation angle definition (typically 1.5 to $2^{\circ}$ ). In this way $\mathrm{A}^{*}$ can be regarded as a multiple of a certain number of dislocation cells within the microstructure. 
The approach of dividing the microstructure into LMRs and HMRs is particularly useful for investigations of microstructural changes occurring during annealing, as the partitioning provides a basis for analyzing a spatial variation in the annealing behavior. Some questions that can be posed are: (i) do nuclei form preferentially in the LMRs or HMRs, or at the interfaces between these regions? (ii) does coarsening occur in a more uniform manner in either the HMRs or the LMRs? and (iii) is the microstructural response during annealing similar for different materials when the LMRs and HMRs are analyzed separately? Such investigations can be carried out either using a direct sequential mapping-annealing-mapping approach (investigation of the same surface before and after annealing) or in a more statistical manner by comparing the LMR and HMR fractions of deformed and partially recrystallized samples. As an example of the latter case, it has been found that for ECAE-processed copper discontinuous recrystallization takes place preferentially inside the HMRs [9,10]. Moreover, it was found that the $f_{\text {LMR }}$ parameter was better correlated to the variation in recrystallization behavior across the sample thickness than the $f_{\mathrm{HAB}}$ parameter [9]. Further details of this investigation are given elsewhere in these proceedings [15].

Table 1: Quantitative description of the microstructure shown in Fig. 1 based on the partitioning into LMRs and HMRs

\begin{tabular}{lccc}
\hline Subset & Area fraction $[\%]$ & $\mathrm{f}_{\mathrm{HAB}}\left(>2^{\mathrm{o}} />15^{\circ}\right)[\%]$ & $\theta_{\mathrm{av}}\left(>2^{\mathrm{o}}\right)\left[{ }^{\circ}\right]$ \\
\hline LMR & 21 & 2 & 5 \\
HMR & 79 & 68 & 27 \\
\hline
\end{tabular}

(a)

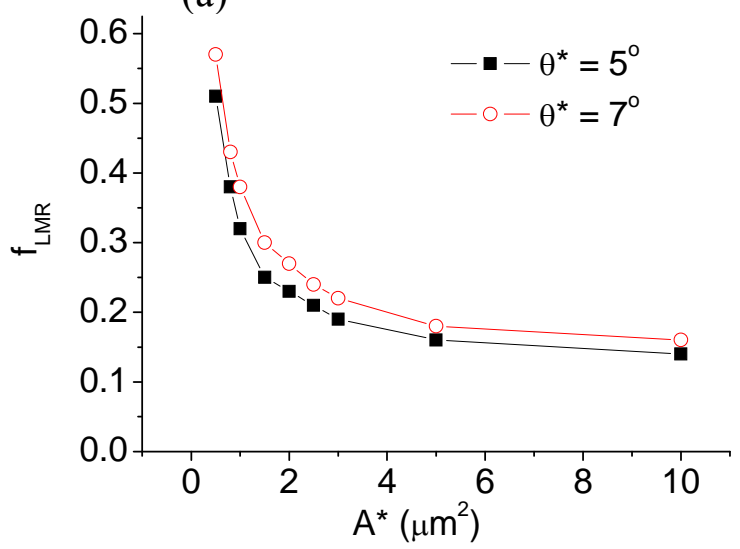

(b)

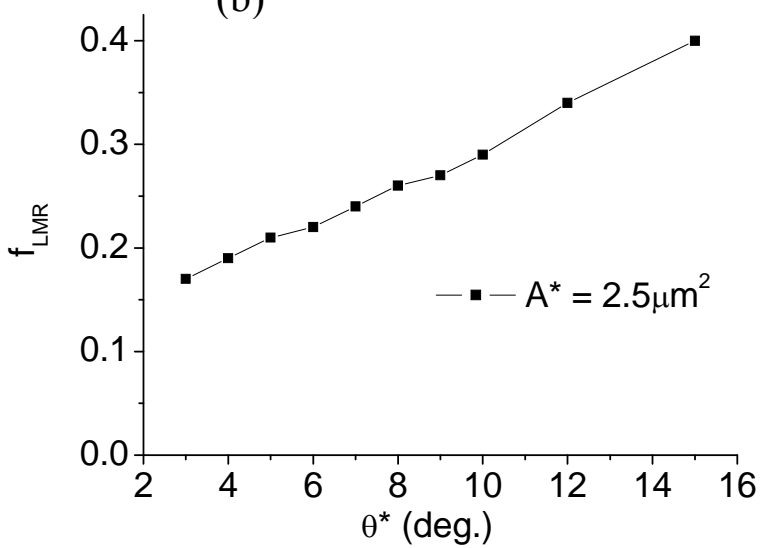

Fig. 3: Variation of $f_{L M R}$ for the map shown in Fig. 1: (a) as a function of $A^{*}$ for fixed values of $\theta^{*}$ of $5^{\circ}$ and $7^{\circ}$; (b) as a function of $\theta^{*}$ for a fixed value of $A^{*}=2.5 \mu \mathrm{m}^{2}$.

\section{Characterization of continuous/discontinuous recrystallization}

A topic that is closely related to the issue of heterogeneity in microstructural refinement is the question of whether annealing of a given sample results in classical discontinuous recrystallization or in a process of continuous microstructural coarsening. Whilst a strong change in texture during annealing is reliable signal of a discontinuous recrystallization process, the converse is not true, i.e. in some highly deformed materials discontinuous recrystallization can occur without a large change in the texture [11,16]. For this reason investigations into this area are best addressed by direct examination of the microstructure. More specifically, the question of interest is whether or not it is possible to identify finely distributed, but nevertheless localized, coarsening during annealing.

The EBSD technique is well suited for such investigations in that orientation data can be collected over large sample areas so that a representative description of the changes occurring during annealing can be obtained. The angular and spatial resolution limitations of the EBSD technique nevertheless place some limitations on the way in which the data can be analyzed. 
One approach that can be taken is to focus on the largest grains/dislocation cells and to ask the question of whether the number of such large grains is consistent with a continuous coarsening process. This approach requires a definition of what constitutes a "large" grain. If we assume that the distribution of dislocation cell sizes in a heavily deformed sample has a known form (e.g. either log-normal or Rayleigh distribution of cell diameters), then the characteristic properties of the distribution can be used as a test for a deviation in the upper tail of the distribution of observed grain sizes. It has been proposed that the mode of the distribution is a good parameter for this purpose [11], as the mode of a distribution is not affected by changes in the tail of the distribution. For example, for a ideal Rayleigh distribution of grain sizes the mode occurs at a value of $\mathrm{d}_{\text {mode }} \approx 0.8 \mathrm{~d}_{\mathrm{av}}$, and only $1.1 \%$ by number of grains (approximately $6 \%$ by area) will have sizes greater than $3 \mathrm{~d}_{\text {mode }}$. Consequently, if a microstructure developed after a short annealing is found to have a significantly larger number of grains with size greater than $3 \mathrm{~d}_{\text {mode }}$ then this can be taken as an indication of a distributed, but locally non-uniform process.

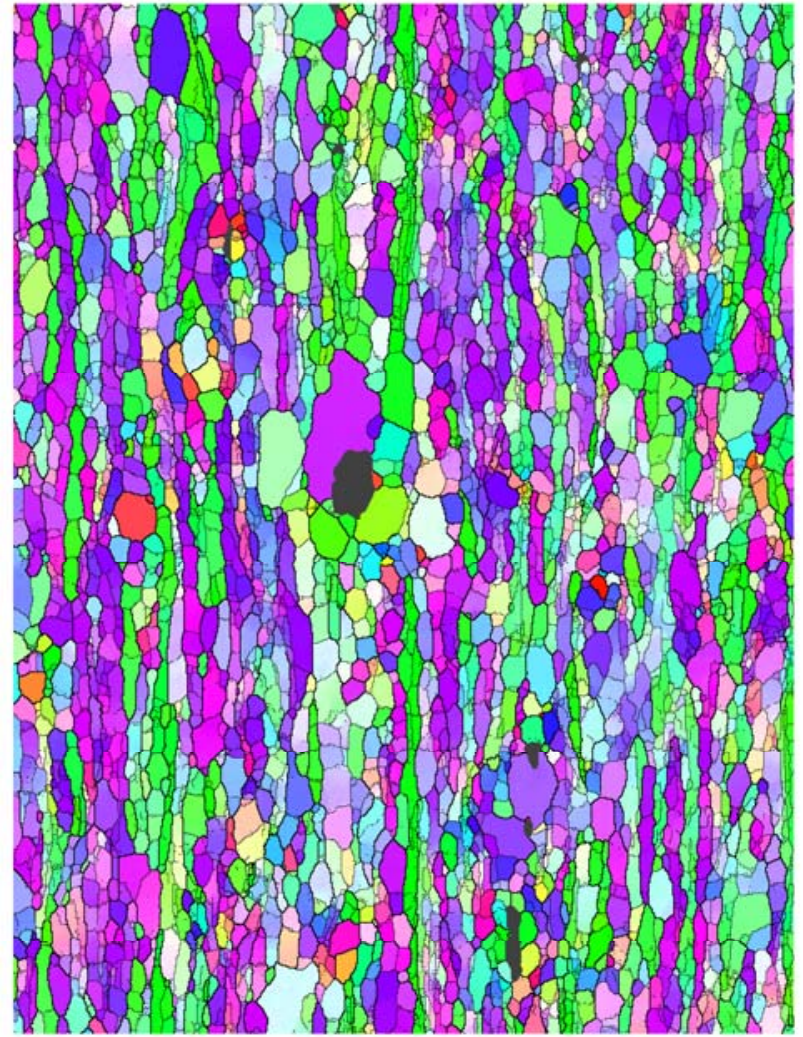

(a)

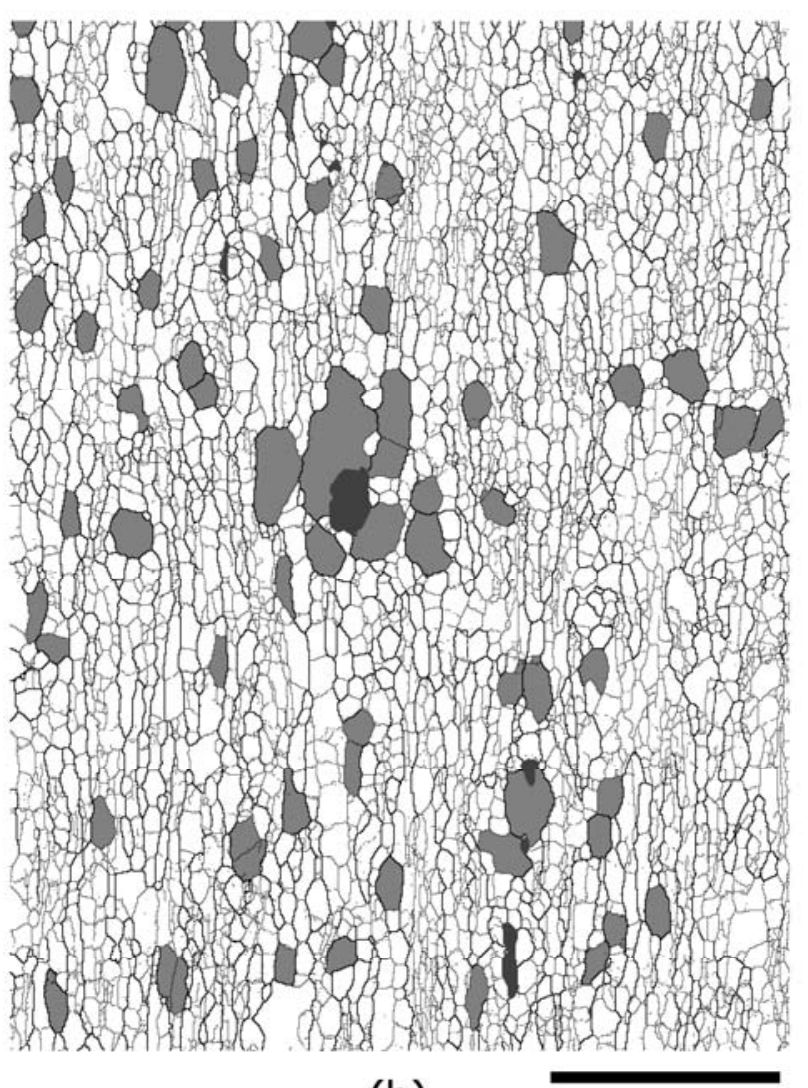

(b)

$20 \mu \mathrm{m}$

Fig. 4: EBSD data (longitudinal section) for a sample of $\mathrm{Al}(99.5 \%)$ cold-rolled to strain $\varepsilon_{\mathrm{vM}}=6.4$ and annealed at $300^{\circ} \mathrm{C}$ for 3 minutes: (a) inverse pole figure coloring (color in online version); (b) subset of detected grains with a size greater than $3 \mathrm{~d}_{\text {mode }}$ (shaded in light grey). In both maps misorientations of $>1^{\circ}$ and $>15^{\circ}$ are shown as thin and thick black lines, respectively. The rolling direction is perpendicular to the scale bar. The dark grey regions correspond to particles where indexing was not possible.

One problem with this approach is that in order to obtain $d_{\text {mode }}$ it is necessary to bin the data. Ideally a bin size should be chosen that gives a good shape to the distribution, but this is complicated by the fact that a low value of $\theta^{*}$ should be used for grain detection in order to identify as many individual dislocation cells as possible, and low values of $\theta^{*}$ also result in the introduction of artificial small "grains", which can distort the shape of the lower end of the grain size distribution. In practice therefore it is necessary to impose some lower cut-off on the grain size and 
then to choose a bin size that in the ideal case places the mode of the distribution in at least the second full bin.

An example of the application of this method is given in Fig. 4a, showing an EBSD orientation map from a sample of $\mathrm{Al}$ deformed to a strain of $\varepsilon_{\mathrm{vM}}=6.4$ by cold-rolling and then annealed at $300^{\circ} \mathrm{C}$ for 3 minutes. For this map grain detection was first carried out using a boundary misorientation definition of $1^{\circ}$. The mode was then determined from the distribution of equivalent circle diameters (note that detected "grains" with an area of less than 3 pixels were ignored in order to limit the effects of orientation noise in the data) using a bin size of $0.3 \mu \mathrm{m}$ as $\approx 0.875 \mu \mathrm{m}$. A subset of grains was then identified with average linear size $>2.65 \mu \mathrm{m}$. Some of these large grains were observed to clearly contain low angle boundaries and therefore represent clusters of dislocation cells. Therefore an additional step of selecting only those grains with an average internal misorientation of less than $0.7^{\circ}$ was applied. The subset of grains finally identified is shown in Fig. $4 \mathrm{~b}$. In this case the subset represents $11 \%$ of the map area, and therefore suggests locally nonuniform restoration processes during annealing.

The method is still limited by the extent to which the low angle dislocation boundaries in the EBSD data can be identified, although because the mode of the distribution is used rather than the mean value, the results are not affected greatly if a part of the lower tail of the distribution is missing. One possibility to improve the method would be to combine the mode-based analysis with the approach suggested by Barou et al. [17] for detection of the boundary structure based on image analysis of a band contrast map, although this may not be suitable for all metals. It is believed that methods for identifying the locations in a microstructure where locally finely distributed but nevertheless non-uniform coarsening may be taking place are useful, particularly for understanding the development of boundary curvature that accompanies the transition from a highly deformed microstructure during the early stages of annealing.

\section{Summary and conclusions}

Metals deformed to large plastic strains typically exhibit a heterogeneity in the refinement of the microstructure by high angle boundaries. In order to characterize this heterogeneity, it is suggested that microstructure can be partitioned into regions that contain predominantly only low angle misorientations and regions that contain predominantly high angle misorientations. Based on this partitioning the parameter $f_{\mathrm{LMR}}$, representing the area fraction of the low misorientation regions can be determined. This parameter has some advantages over the more commonly used fraction of HABs $\left(f_{\mathrm{HAB}}\right)$ parameter, particularly in that it is insensitive to missing information about very low angle boundaries in EBSD data, arising from the limited angular resolution of this technique. The parameter also allows a length scale for the pattern of heterogeneity in refinement by high angle boundaries to be determined.

For the investigation of microstructural coarsening during the annealing of metals deformed to high strains a procedure based on identification of dislocation cells/grains with a size greater than a fixed multiple of the mode of the size distribution is suggested. This procedure allows identification of locally non-uniform but nevertheless highly distributed coarsening events, and is important therefore for investigations of the early stages of microstructural restoration during annealing of heavily deformed materials.

\section{Acknowledgements}

The authors gratefully acknowledge support from the Danish National Research Foundation and the National Natural Science Foundation of China (Grant No. 50911130230) for the Danish-Chinese Center for Nanometals, within which this work was performed. 


\section{References}

[1] R.Z. Valiev, R.K. Islamgaliev and I.V. Alexandrov: Prog. Mater. Sci., Vol. 45 (2000), p. 103.

[2] N. Tsuji, Y. Ito, Y. Saito and Y. Minamino: Scripta Mater., Vol. 46 (2002), p. 893.

[3] A. Gholinia, P.B. Prangnell and M.V. Markushev: Acta Mater., Vol. 48 (2000), p. 1115.

[4] N. Tsuji, N. Kamikawa, R.Ueji, N.Takata, H.Koyama and D.Terada: ISIJ International, Vol. 48 (2008) p. 1114.

[5] R.D. Doherty, D.A. Hughes, F.J. Humphreys, J.J. Jonas, D. Juul Jensen, M.E. Kassner, W.E. King, T.R. McNelley, H.J. McQueen, A.D. Rollet: Mater. Sci. Eng. A, Vol. 238 (1997), p. 219.

[6] H. Jazaeri and F.J. Humphreys: Acta Mater., Vol 52. (2004), p. 3251.

[7] A. Oscarsson, H-E. Ekström, B. Hutchinson, Mater. Sci. Forum, Vols 113-115 (1993), p. 177.

[8] H.W. Zhang, X. Huang and N. Hansen: Acta Mater., Vol. 56 (2008), p. 5451.

[9] O.V. Mishin and J.R. Bowen: Metall. Mater. Trans. A, Vol. 40A (2009), p. 1684.

[10] O.V. Mishin and A. Godfrey: Metall. Mater. Trans. A, Vol. 39A (2008), p. 2923.

[11] W.Q. Cao, A. Godfrey, N. Hansen and Q. Liu: Metall. Mater. Trans. A, Vol. 40A (2009), p. 204.

[12] A. Godfrey, O.V. Mishin, J. Bowen and Q. Liu, in: Proc. 30th Risø International Symposium on Materials Science, edited by J.C. Grivel et al., Risø-DTU, Roskilde, p.31 (2009).

[13] O.V. Mishin A. Godfrey and L. Östensson: Metall. Mater. Trans. A, Vol. 37A (2006), p. 489.

[14]A. Godfrey: Scripta Mater. Vol. 50 (2004), p. 1097.

[15] O.V. Mishin, J.R. Bowen and A. Godfrey, Mater. Sci. Forum, (2010), in press.

[16] O.V. Mishin, D. Juul Jensen and N. Hansen: Metall. Mater. Trans. A, Vol. 41A (2010), p. 2936.

[17] F. Barou, C. Maurice, J-M. Feppon and J. Driver: Int. J. Mater. Res., Vol. 100 (2009), p. 516. 